Case Report

\title{
Permanent pacemaker associated infective endocarditis caused by a member of the HACEK group of organisms: a case report
}

\author{
G Lakshy ${ }^{1}$, SK Jayatilleka², SRP Kottegoda ${ }^{2}$ \\ Sri Lankan Journal of Infectious Diseases 2018 Vol.8 (1):40-45 \\ DOI: http://dx.doi.org/10.4038/sljid.v8i1.8143
}

\begin{abstract}
The HACEK group of organisms have fastidious growth requirements and usually present as culture negative endocarditis. Probable Aggregatibacter aphrophilus has been isolated in a routine microbiology laboratory in a patient with a permanent pacemaker with echocardiographic features suggestive of lead endocarditis. Device removal, though recommended, was not carried out for clinical and economic reasons. The patient was successfully treated with antibiotics including intravenous ceftriaxone for the duration of two weeks plus oral cefixime for further 4 weeks together with regular and extended follow-up for more than one year while the pacemaker and the leads remained in-situ.
\end{abstract}

Keywords: Pacemaker endocarditis, HACEK organisms, Haemophilus spp., Aggregatibacter spp.

\section{Introduction}

Infection remains a major complication of implanted cardiovascular devices even with advanced techniques such as improvements in device manufacture and minimal access surgery. ${ }^{1}$ Endocarditis is the most severe form of infection which is associated with significant morbidity, mortality and financial cost. ${ }^{1}$ Endocarditis due to the HACEK group of organisms, in particular Haemophilus parainfluenzae and Aggregatibacter aphrophilus have been reported rarely, and account for up to $5 \%$ of infective endocarditis. ${ }^{1,3-5}$ The HACEK group includes Haemophilus spp., Aggregatibacter spp., Cardiobacterium spp., Eikenella spp. and Kingella spp. They have fastidious growth requirements and usually present as culture negative endocarditis ${ }^{1,3-5}$.

\section{Case report}

A 22-year-old university student was admitted with a history of intermittent fever for 2 weeks which was associated with chills, myalgia, loss of appetite, nausea and vomiting. He had a permanent pacemaker (PPM) implantation two years back for vasovagal syncope of cardioinhibitory type. Transthoracic echocardiogram (TTE) was performed soon after admission which showed an echogenic particle attached to the right atrial lead. Transoesophageal echocardiogram (TOE) showed an echogenic oscillating mass attached to the right atrial lead which was suggestive of PPM lead endocarditis. The patient was diagnosed as having

${ }^{1}$ Postgraduate Institute of Medicine, University of Colombo, Sri Lanka.

${ }^{2}$ Sri Jayewardenepura General Hospital, Nugegoda, Sri Lanka.

Address for correspondence: Dr Lakshy Guruparan, Sri Jayewardenepura General Hospital, Nugegoda, Sri

Lanka. Telephone: +94776337430 Email:lakshys@gmail.com D http://orcid.org/0000-0001-5271-6914

Received 11 July 2017 and revised version accepted 27 March 2018

(c) $(7)$

This an open-access article distributed under the terms of the Creative Commons Attribution License, which permits unrestricted use, distribution, and reproduction in any medium, provided the original author and source are credited. 
probable lead endocarditis and intravenous ceftriaxone $2 \mathrm{~g}$ daily and vancomycin $1 \mathrm{~g} 12$ hourly were started empirically after taking 3 sets of blood cultures. The need to remove the PPM was also considered.

The blood cultures signalled positive in 56 and 76 hours duration. Gram negative bacilli were seen in the direct Gram stain smears of the blood culture broths. However, they did not grow on blood and chocolate agar plates incubated in 5-10\% $\mathrm{CO}_{2}$ (candle jar) and MacConkey agar incubated in air for 24 hours. The culture plates were re-incubated for a further 7 days. Meanwhile the blood culture broths were subcultured again on blood and chocolate agar and incubated in an anaerobic environment. A powdery growth at 24 hours on the anaerobic plates was Gram stained and showed slender Gram negative bacilli. Similar growth was also seen on the aerobic plates after 7-8 days of incubation. The isolate was sub cultured on blood agar with Staphylococcus aureus stabs and satellitism (growth around the stabs) was noted. When grown on Muller Hinton agar with $\mathrm{X}, \mathrm{V}$ and $\mathrm{XV}$ factor discs, there was growth around the $\mathrm{V}$ and $\mathrm{XV}$ factor discs, but not around the $\mathrm{X}$ factor disc. The isolate was non-haemolytic and catalase positive. It was identified as probable Aggregatibacter aphrophilus. ${ }^{1}$

With the identification of the blood culture isolate as probable Aggregatibacter aphrophilus, vancomycin was omitted, while continuing ceftriaxone. Although removal of the atrial lead was recommended, it was not carried out due to the ongoing need for the device, possible complications such as embolization with removal and for economic reasons. The patient was treated with IV ceftriaxone $2 \mathrm{~g}$ daily for the duration of two weeks while the device was insitu. The patient's clinical condition improved with the parenteral antimicrobial therapy and the repeated transoesophageal echocardiogram (TOE) showed reduction in the size of the right atrial lead thickening. Treatment was continued with oral cefixime $400 \mathrm{mg}$ daily for further 4 weeks. He was reviewed weekly with CRP levels and a 2D Echo cardiogram was carried out on completion of therapy. In addition, instructions were given regarding oral hygiene and risk of recurrences or relapse and to return if symptoms recur. The patient remained clinically well with satisfactory parameters at the one year follow up.

The timeline of the patient's clinical course

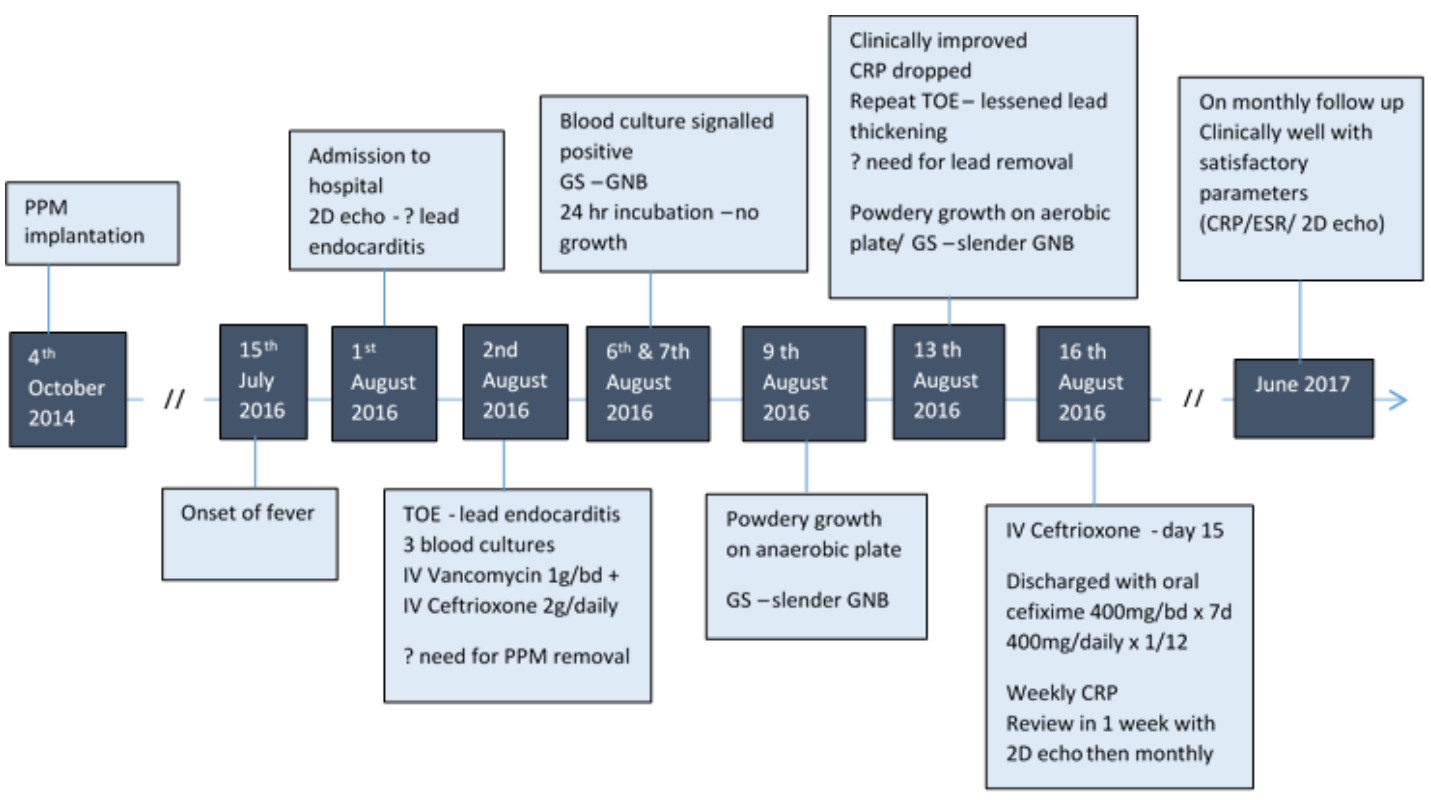




\section{Discussion}

Cardiac implantable electronic device (CIED) associated infections can be early onset (within the first 6 months since implantation) or late onset (> 6 months). ${ }^{1}$ This patient had a late onset infection which is usually due to haematogenous seeding of organisms onto cardiac devices. Gram positive organisms such as Staphylococcus spp. are known to be the commonest pathogens in late onset infection. ${ }^{1,2}$ However, a Gram negative pathogen belonging to the HACEK group, a rare cause, was isolated from this patient.

CIED associated infections have been associated with some specific risk factors related to the patient, device or procedure, such as older age, co-morbid conditions, tunnelled central venous catheter, chronic anticoagulation, long-term corticosteroid therapy, multiple devicerelated procedures and recent device manipulation. ${ }^{1}$ This patient did not have any of these identified risk factors. However, he did have widespread dental caries.

There were two major criteria ${ }^{3-5}$ to diagnose endocarditis in this patient. Blood cultures were positive for a typical microorganism (HACEK group) consistent with endocarditis from two separate blood cultures and the evidence of endocardial involvement with the transoesophageal echocardiogram showing an oscillating intra cardiac mass on implanted material without an alternative anatomic explanation. The patient also had fever and predisposition for endocarditis due to the prosthetic device.

Isolation of the pathogen from the blood culture was very challenging. Though the direct smear showed Gram negative bacilli, they could not be isolated easily. The isolate took 7-8 days to grow when incubated on blood/chocolate agar plates in the candle jar but with the anaerobic pack it grew rapidly. This could have been due to inadequate $\mathrm{CO}_{2}$ within the candle jar. The anaerobic pack releases $7-15 \% \mathrm{CO}_{2}$ within 24 hours.

Identification of the HACEK group of organisms can be confirmed by conventional/commercial biochemical tests or by automated systems such as matrix-assisted laser desorption/ionization time-of-flight mass spectrometry (MALDI-TOF MS) or by molecular tests such as PCR. ${ }^{6}$ In the absence of facilities for identification, the isolate was designated as probable Aggregatibacter aphrophilus (former Haemophilus paraphrophilus) based on Gram stain appearance of pleomorphic gram negative bacilli and the basic cultural factors such as absence of haemolysis, $\mathrm{V}$ factor requirement and $\mathrm{CO}_{2}$ dependence. ${ }^{1,6} A$. aphrophilus, formerly called Haemophilus aphrophilus and $H$. paraphrophilus has been reclassified as a single species on the basis of multi locus sequence analysis. ${ }^{7,8} \mathrm{~A}$ aphrophilus includes V-factor dependent and V-factor independent isolates. The V factor dependent phenotype is caused by partial genetic deletion which is naturally reversible. Both $\mathrm{V}$ factor dependent and independent phenotypes were homogenous by 16S rRNA gene sequences. ${ }^{8}$

A. aphrophilus is a known commensal of the human oral cavity and pharynx and may enter the blood stream with dental procedures. This patient did not undergo any dental treatment in the recent past, but had severe dental caries, which could have caused transient bacteraemia during day to day activities such as brushing or chewing resulting in infection of the prosthetic device. This is postulated to be a possible mechanism for transient bacteraemia when endocarditis prevention is concerned. ${ }^{1}$ 
Endocarditis caused by $A$. aphrophilus has been reported worldwide, including valvular ${ }^{9-11}$ and pacemaker endocarditis. ${ }^{12}$ It is also reported to cause several other infections, particularly abscesses, such as cerebral abscess, liver abscess, lung abscess and empyema, septic arthritis, osteomyelitis and facial cellulitis. ${ }^{1}$ The clinical course of Aggregatibacter spp. endocarditis tends to be subacute and embolization is seen commonly. ${ }^{1}$ The patient seen by us had an uncomplicated clinical course.

The HACEK group of organisms should be suspected when there is a strong clinical suspicion of endocarditis and negative blood cultures. As they are slow growing, incubation of blood cultures for 2 weeks is recommended. ${ }^{1,3}$ However in this case, the blood culture became positive within 5 days of incubation (56 hours and 76 hours) which is compatible with studies that indicate the HACEK group of organisms can be isolated with standard blood culture incubation periods using conventional automated blood culture systems. ${ }^{1}$

The HACEK group of microorganisms were consistently susceptible to ampicillin earlier, but ampicillin resistance is being reported with increased frequency. ${ }^{2}$ Penicillin and ampicillin should therefore not be used to treat patients with endocarditis as the slow growth of these organisms makes it difficult to perform susceptibility tests. Almost all strains of the HACEK group are susceptible to ceftriaxone or other third- or fourth-generation cephalosporins ${ }^{3,13}$ and hence ceftriaxone has commonly been used to treat HACEK endocarditis. ${ }^{1,3-5}$ This group is usually susceptible in vitro to fluoroquinolones ${ }^{3,13}$. A fluoroquinolone (ciprofloxacin, levofloxacin, or moxifloxacin) may be considered as an alternative agent in patients unable to tolerate third- or fourth-generation cephalosporin therapy. ${ }^{3-5}$

Although the patient was successfully treated without relapse, there were several limitations in the management of this patient with regard to adhering to evidence based therapeutic guidelines.

Intravenous antibiotics are recommended for the whole duration of therapy of infective endocarditis. There is insufficient evidence to continue with an oral regime as was done with this patient. $^{2-5}$ Rapid discharge on outpatient parenteral antibiotic therapy (OPAT) is recommended ${ }^{3-5}$ to reduce duration of hospital stay. This patient was well enough to resume his academic activities. However, the healthcare system in Sri Lanka is still not equipped with facilities for OPAT. Oral therapy was therefore instituted in this patient.

Guidelines recommend complete device and lead removal when there is definite infection such as lead endocarditis. ${ }^{2}$ However in this patient, the cardiac device was retained due to the ongoing need for the device, possible complications such as embolization with removal and for economic reasons. On periodical review of this patient over a year with echocardiogram and biomarkers, the patient remained clinically well without relapse. The patient was advised on oral hygiene and to be vigilant about the risk of cardiac device associated infection in the future.

\section{Conclusions}

There are possibilities to isolate fastidious organisms in a routine conventional microbiology laboratory with limited resources if conditions such as prolonged incubation and adequate $\mathrm{CO}_{2}$ levels are provided. Limitations also result in compromised care as seen in this patient, with switch to an oral antibiotic and non-removal of the infected device. The need to establish outpatient parenteral antimicrobial therapy (OPAT) in our healthcare system for patients on 
long term antibiotics who are clinically well and able to pursue their usual activities is demonstrated with this patient.

\section{Acknowledgments}

We would like to acknowledge the patient for consenting to report this case.

\section{Conflict of Interest}

The authors do not have any conflicts of interest regarding this case report.

\section{References}

1. Bennett JE, Dolin R, Blaser MJ (eds). Mandell, Douglas and Bennett's Principles and Practice of Infectious disease $\left(8^{\text {th }}\right.$ ed). Philadelphia: Elsevier Saunders, 2015.

2. Baddour LM, Epstein AE, Erickson CC, et al. Update on cardiovascular implantable electronic device infections and their management: A scientific statement from the American heart association. Circulation 2010; 121:458-477.

doi: 10.1161/CIRCULATIONAHA.109.192665

3. Baddour LM, Wilson WR, Bayer AS, et al. Infective endocarditis in adults: diagnosis, antimicrobial therapy and management of complications. A Scientific Statement for Healthcare Professionals from the American Heart Association. Circulation. 2015; 132:00-00.

doi: 10.1161/CIR.0000000000000296.

4. Gould FK, Denning DW, Elliott TSJ, et al. Guidelines for the diagnosis and antibiotic treatment of endocarditis in adults: a report of the Working Party of the British Society for Antimicrobial Chemotherapy. Journal of Antimicrobial Chemotherapy 2012; 67:269-289

doi:10.1093/jac/dkr450

5. Habib G, Lancellotti P, Antunes MJ, et al. 2015 ESC Guidelines for the management of infective endocarditis. European Heart Journal 2015; ehv319.

doi:10.1093/eurheartj/ehv319.

6. Health Protection Agency. Identification of Haemophilus species and the HACEK Group of Organisms. UK Standards for Microbiology Investigations 2011; ID 12 Issue 2.2. doi: http://www.hpa.org.uk/SMI/pdf.

7. Lauritsen NN. Classification, identification and clinical significance of Haemophilus and Aggrigatibacter species with host specificity for humans. Clinical Microbiology Reviews 2014; 27(2):214-240. doi: http://dx.doi.org/10.1128/CMR.00103-13

8. Lauritsen NN, Kilian M. Reclassification of Actinobacillus actinomycetemcomitans, Haemophilus aphrophilus, Haemophilus paraphrophilus and Haemophilus segnis as Aggregatibacter actinomycetemcomitans gen. nov., comb. nov., Aggregatibacter aphrophilus comb. nov. and Aggregatibacter segnis comb. nov., and emended description of Aggregatibacter aphrophilus to include V factor-dependent and V factor-independent isolates. International Journal of Systematic and Evolutionary Microbiology 2006; 56:2135-2146. doi: 10.1099/ijs.0.64207-0

9. Chambers ST, Murdoch D, Morris A, et al. HACEK Infective Endocarditis: characteristics and outcomes from a large, multi-national cohort. PLOS ONE 2013; 8(5):e63181. doi: 10.1371/journal.pone.0063181

10. Sood S. Infective Endocarditis by Aggregatibacter paraphrophilus: Case report and literature review. Journal of Clinical and Diagnostic Research 2013; 7(11):2577-2578. doi: 10.7860/JCDR/2013/5794.3617

11. Broa AL, Cruz I, Cotrim C,et al. Aggregatibacter aphrophilus aortic valve infective endocarditis. Infectio 2016. doi: http://dx.doi.org/10.22354/in.v21i2.656

12. Patel SR, Patel NH, Borah A, et al. Aggregatibacter aphrophilus pacemaker endocarditis: a case report. BMC Research Notes 2014; 7:885.

doi: http://www.biomedcentral.com/1756-0500/7/885

13. Coburn B, Toye B, Rawte P, et al. Antimicrobial susceptibilities of clinical isolates of HACEK organisms. Antimicrobial Agents and Chemotherapy 2013; 57 (4): 1989-1991 
doi:10.1128/AAC.00111-13

14. Yew HS, Chambers ST, Roberts SA, et al. Association between HACEK bacteraemia and endocarditis. Journal of Medical Microbiology 2014; 63(6): 892-895.

doi: 10.1099/jmm.0.070060-0 